\title{
Manifestaciones dermatológicas y enfermedades concomitantes en pacientes con acromegalia o gigantismo
}

Dermatologic manifestations and comorbidities in patients with acromegaly or gigantism

\section{Wilson Galvis-Franco', Humberto Ignacio Franco ${ }^{2}$, Alín Abreu-Lomba ${ }^{3}$, Consuelo Vélez- Álvarez ${ }^{4}$}

1. Médico dermatólogo; docente, Universidad de Antioquia, Medellín

2. Médico endocrinólogo; docente, Universidad de Caldas, Manizales

3. Médico endocrinólogo, Centro Médico Imbanaco, Cali

4. Enfermera, epidemióloga, Ph. D. en Salud Pública; docente, Universidad de Caldas, Manizales, Colombia

\section{RESUMEN}

Introducción: La acromegalia y el gigantismo son enfermedades causadas por la producción excesiva de hormona de crecimiento. En la mayoría de los pacientes que presentan estas enfermedades se describen cambios cutáneos, al igual que enfermedades concomitantes que aumentan su morbimortalidad.

Objetivo: Describir las manifestaciones dermatológicas y las enfermedades concomitantes, en pacientes con acromegalia o gigantismo.

Métodos: Se desarrolló un estudio descriptivo y transversal, en el cual se evaluaron y describieron las características dermatológicas y enfermedades sistémicas asociadas, en pacientes con acromegalia o gigantismo valorados en la consulta de endocrinología de las ciudades de Manizales y Armenia, en el periodo de abril y mayo de 2013 .

Resultados: Se describen los hallazgos dermatológicos y comorbilidades en 20 pacientes con acromegalia y 2 con gigantismo, todos ellos con adenoma hipofisiario. Se encontró engrosamiento de la piel y el cuero cabelludo en $77.3 \%$ de los casos, agrandamiento distal de extremidades (acral) en 95.5\%, agrandamiento de nariz en $81.8 \%$, agrandamiento de orejas en $27.3 \%$, agrandamiento de labios en $50 \%$, macroglosia en $54.5 \%$, prominencia frontal en $63.6 \%$, y prognatismo y mala oclusión en $68.2 \%$, entre otras manifestaciones clínicas. Se encontraron enfermedades sistémicas asociadas en $63.6 \%$, principalmente, hipertensión arterial (50\%), diabetes (27.3\%) y dislipidemia (45.5\%).

Conclusión: La población analizada en este estudio no difiere de otras descritas en la literatura médica mundial. Sin embargo, los hallazgos corroboran la gran frecuencia de manifestaciones dermatológicas y comorbilidades y su importancia al momento del enfoque clínico, ya que permiten sospechar la enfermedad y pueden llevar a un diagnóstico temprano que impacte positivamente en su morbimortalidad.

PAlABRAS CLAVE: acromegalia, gigantismo, manifestaciones cutáneas, piel.

\section{Correspondencia:}

Wilson Galvis Franco

Email:

wilsongalvisf@yahoo.com

Recibido: 09/05/16

Aceptado: 25/10/18

\section{Conflictos de interés:}

No se reportan conflictos de interés.

Financiación:

Ninguna. 


\section{SUMMARY}

Introduction: Acromegaly and gigantism are diseases caused by an excessive production of growth hormone. Most of patients who suffer these conditions are affected with skin changes, as well as comorbidities increasing their morbidity and mortality.

Objective: To describe the dermatological manifestations and associated comorbidities in patients with acromegaly and gigantism.

Methods: A descriptive cross-sectional study was developed, which evaluated and described the dermatological features and systemic diseases associated in patients with acromegaly and gigantism valued in endocrinology in Manizales and Armenia in the the period April to May, 2013.

Results: The dermatological findings and associated comorbidities in 20 patients with acromegaly and 2 with gigantism are described; a pituitary adenoma was demonstrated in all of them. The findings were as follows: thickening of skin and scalp , 77.3\% of patients; acral enlargement, 95.5\%; enlarged nose , 81.8\%; enlarged ears , 27.3\%; enlarged lips, 50\%; macroglossia, 54.5\%; likewise, frontal bossing was found in $63.6 \%$, prognathism and malocclusion in $68.2 \%$, among others. Systemic diseases were associated in $63.6 \%$, mainly hypertension in $50 \%$, diabetes in $27.3 \%$ and dyslipidemia in $45.5 \%$.

Conclusion: The population analyzed in this study does not differ from that described in medical literature; the findings support the high frequency of dermatological manifestations and associated comorbidities in these patients and its importance at the time of clinical approach, allowing to suspect the disease and may lead to an early diagnosis that will impact positively morbidity and mortality.

KEY WORDS: Acromegaly, gigantism, skin manifestations, skin.

\section{INTRODUCCIÓN}

La acromegalia o gigantismo es una enfermedad causada por la producción excesiva de la hormona del crecimiento, debido a un adenoma hipofisiario en más del $90 \%$ de los casos. Las causas extrapituitarias son extremadamente raras e incluyen tumores pancreáticos y pulmonares, entre otros. Esta enfermedad se denomina acromegalia cuando afecta a los adultos y gigantismo cuando los afectados son niños. Es una rara enfermedad con una prevalencia calculada de 40 a 70 casos por millón de habitantes y una incidencia anual de 3 a 4 casos nuevos por millón de habitantes. No obstante, otros estudios, como uno realizado en Bélgica, sugieren que los adenomas pituitarios pueden ser más prevalentes, de tal manera que la prevalencia de la acromegalia podría ser de alrededor de 100 a 130 casos por millón ${ }^{(1-4)}$.

La enfermedad se caracteriza por un desfiguramiento somático adquirido y progresivo, principalmente en cara y extremidades, aunque puede afectar otros órganos asociándose con manifestaciones sistémicas que aumentan la morbimortalidad dos a cuatro veces, en comparación con la población general.

$\mathrm{Su}$ comienzo insidioso hace que el diagnóstico sea tardío, con una media reportada de 9 años de evolución antes de que se lleve a cabo. La enfermedad afecta por igual ambos sexos, con una edad promedio al momento del diagnóstico de 40 años en el sexo masculino y de 45 años en el femenino. En la mayoría de los casos informados, se han descrito cambios cutáneos tem$\operatorname{pranos}^{(5-7)}$.

Hasta donde se sabe, no hay ningún estudio a nivel nacional sobre este tópico, por lo cual se llevó a cabo este trabajo para describir las manifestaciones dermatológicas y las comorbilidades en pacientes con acromegalia o gigantismo, con el propósito de conocer su comportamiento clínico a nivel local.

\section{MATERIALES Y MÉTODOS}

Se desarrolló un estudio descriptivo transversal, en el cual se evaluaron y se describieron las características dermatológicas y las enfermedades sistémicas asociadas con acromegalia o gigantismo, valorados en la consulta de Endocrinología de Manizales y Armenia en el eje cafetero de Colombia, durante el periodo de abril a mayo del 2013.

El criterio de inclusión utilizado fue pacientes con diagnóstico de acromegalia o gigantismo atendidos en la consulta de Endocrinología, que firmaron el consentimiento informado para autorizar la valoración clínica dermatológica; se excluyeron aquellos que no otorgaron el consentimiento informado.

Se hicieron una valoración clínica y un examen físico completos, previo consentimiento informado firmado por el paciente o su acudiente. Se describieron todos los hallazgos dermatológicos y las comorbilidades, y se revisaron los exámenes paraclínicos de cada paciente. También, se tomaron medidas del grosor de la piel en algunas áreas corporales de todos los pacientes, como 
los brazos y los antebrazos, y se compararon con las de un grupo control con características similares, que no padecían la enfermedad; estas mediciones se hicieron con un plicómetro en el mismo sitio en cada persona valorada.

La historia clínica fue diligenciada directamente por el mismo investigador en todas las consultas, para controlar posibles sesgos de observación. Se tomaron fotos de los hallazgos clínicos más relevantes durante la evaluación, previo consentimiento del paciente.

El análisis estadístico de los hallazgos clínicos y exámenes paraclínicos, fue realizado con el programa SPSS $^{\mathrm{TM}}$, versión 15.0, con licencia para la Universidad de Caldas.

\section{RESULTADOS}

Se describen 22 pacientes, 9 de sexo masculino (40.9 $\%)$ y 13 de sexo femenino (59.1\%), con un rango de edad en los pacientes valorados entre 11 y 80 años. Según el estado civil, hubo 9 pacientes casados, 2 separados, 1 divorciado, 9 solteros y 1 viudo. Los estratos socioeconómicos predominantes fueron el 2 y el 3. Se encontraban afiliados al régimen de salud contributivo 21 pacientes (95.5\%) y al subsidiado, 1 (4.5\%).

De los pacientes analizados, 20 tenían diagnóstico de acromegalia y 2 de gigantismo. El tiempo transcurrido entre el diagnóstico y el ingreso al estudio, fue de 55.,7 meses en promedio, con un rango entre 1 y 156 meses. Según las pruebas de laboratorio encontradas en el historial clínico de estos pacientes, en promedio, la concentración sérica de la hormona del crecimiento o somatotropina, fue de $9 ., 1 \mathrm{ng} / \mathrm{ml}$, y la de la somatomedina C o IGF-1 (Insulin-like Growth Factor-1) fue de 655 $\mathrm{ng} / \mathrm{ml}$. Estos datos estaban disponibles en 20 de los 22 pacientes estudiados. Además en 16 pacientes con medición de la prolactina, su valor promedio fue de 641 $\mathrm{ng} / \mathrm{ml}$.

Todos los pacientes presentaban adenoma hipofisiario demostrado por resonancia magnética (RM): macroadenoma en $13(59.1 \%)$ y microadenoma en 9 de ellos (40.9\%).

El tratamiento médico consistió en la administración de lanreotide en 18 pacientes (81.8\%) y de octreotide en $1(4.5 \%)$, y 3 pacientes no recibían tratamiento aún (13.6\%). Además, 11 pacientes (50\%) se sometieron a tratamiento quirúrgico y, 8 (36.4\%), a radioterapia.

Se realizó la valoración clínica completa en todos los pacientes y se hallaron las siguientes alteraciones cutáneas y de tejidos blandos: engrosamiento de piel y cuero cabelludo en 17 pacientes (77.3\%), agrandamiento distal de extremidades (acral) en 21 (95.5\%)

(figuras 1 y 2), agrandamiento de la nariz en 18 (81.8 $\%$, aumento del tamaño de las orejas en 6 (27.3\%),

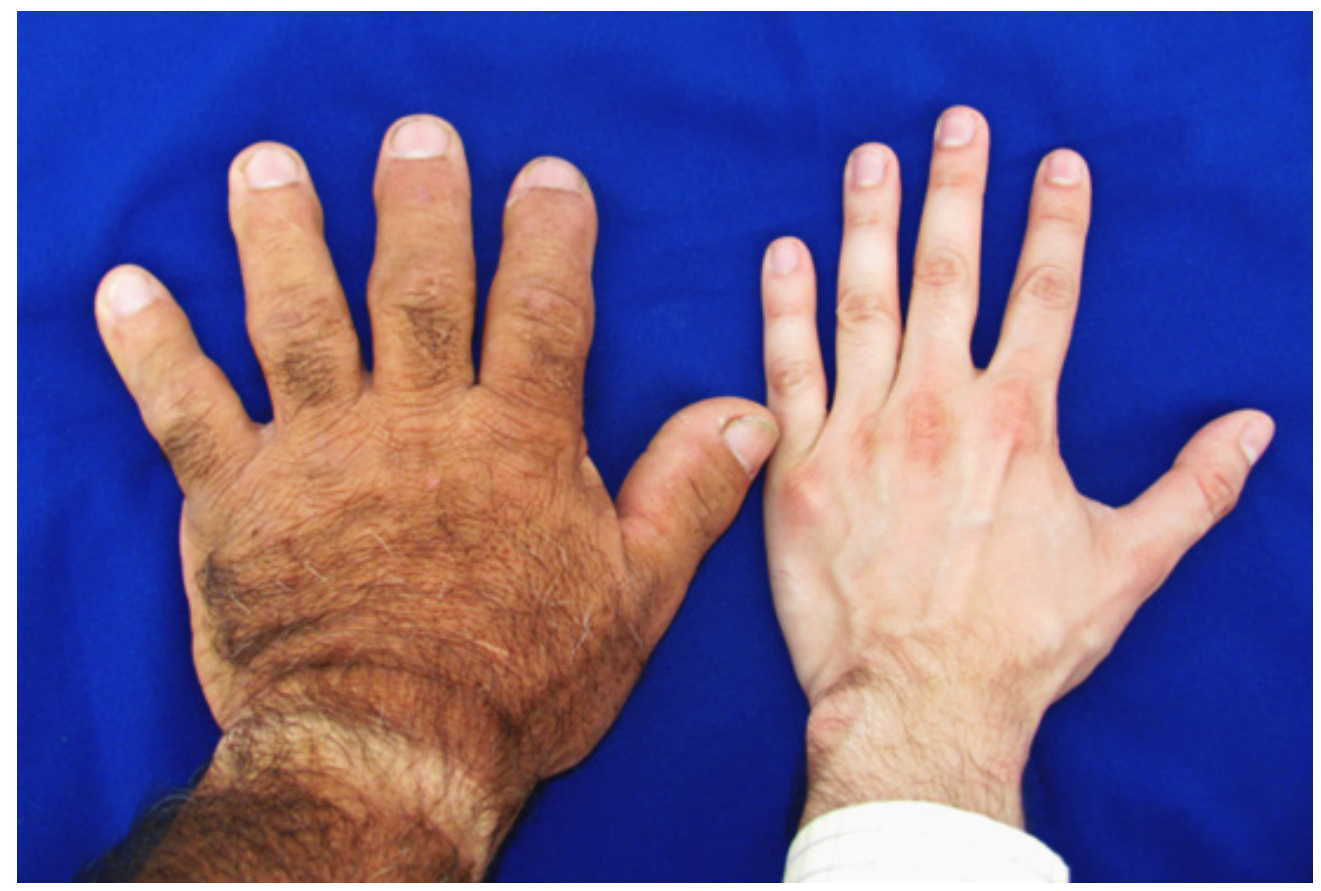

Figura 1. Agrandamiento distal de las extremidades superiores (acral). Foto comparativa con un individuo sin gigantismo 


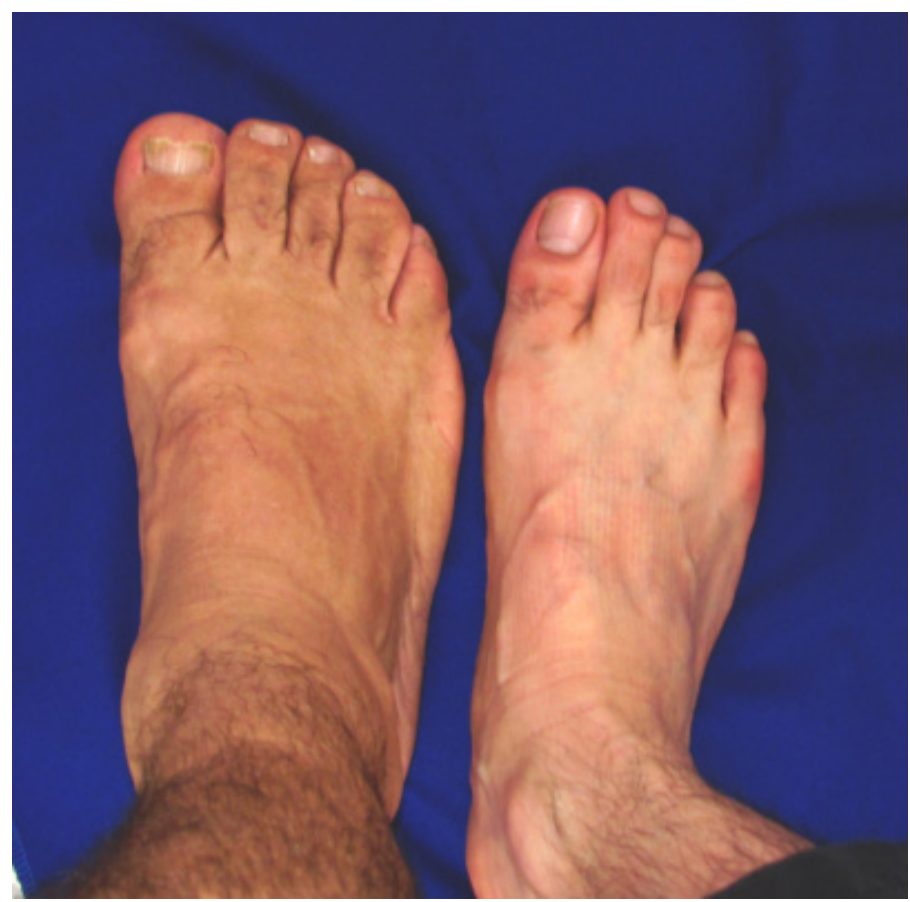

Figura 2. Agrandamiento distal de las extremidades inferiores (acral). Foto comparativa con un individuo sin gigantismo

agrandamiento de los labios en 11 (50 \%) y macroglosia en 12 (54.5\%) (figura 3); además, se observaron acantosis nigricans en 4 pacientes (18.2 \%), fibromas laxos o acrocordones en 16 (72.7 \%), hirsutismo e hipertricosis en 10 ( $45.5 \%)$, hiperhidrosis en 13 (59.1\%), bromhidrosis en 2 (9.1\%), cutis verticis gyrata en $4(18.2 \%)$ (figura 4), y cambios ungulares en 10 (45.5\%), prominencia frontal en $14(63.6 \%)$, prognatismo y mala oclusión en 15 (68.2 \%) y alteraciones óseas en 14 (63.6 $\%)$. Otras alteraciones dermatológicas encontradas en menor proporción fueron queratosis seborreicas, queratosis actínicas, quiste epidérmico y carcinoma basocelular (tabla 1).

Las enfermedades sistémicas concomitantes se encontraron en 14 de los 22 pacientes analizados (63.6\%), y fueron: hipertensión arterial sistémica en 11 (50\%), diabetes mellitus en 6 (27.3\%), dislipidemia en 10 (45.5 $\%)$, hipotiroidismo en 5 (22.7\%), cardiopatía en 4 (18.2 $\%$, artropatía en $4(18.2 \%)$ y alteraciones intestinales en $5(22.7 \%)$, con pólipos intestinales en $3(13.6 \%)$ de ellos; ningún paciente presentó cáncer de colon.

Se encontraron también acroparestesias y síndrome del túnel carpiano en 12 pacientes (54.5\%). Diez pacientes refirieron cefalea (45.5\%); 19 con alteraciones visuales (86.4\%); 8 (36.4\%) cambios en la voz; 14 (63.6 $\%)$ alteraciones respiratorias y del sueño, con apnea del sueño en $5(22.7 \%)$; 11 (50 \%) somnolencia y fatiga; $12(54.5 \%)$ alteraciones sexuales; 12 (54.5\%) disminución de la libido; y 3 (13.6\%) impotencia (tabla 2).

La valoración clínica incluyó mediciones del grosor de la piel, con un plicómetro, en diferentes zonas del cuerpo. una medición en el área posterior del brazo 
Manifestaciones dermatológicas y enfermedades

concomitantes en pacientes con acromegalia o gigantismo

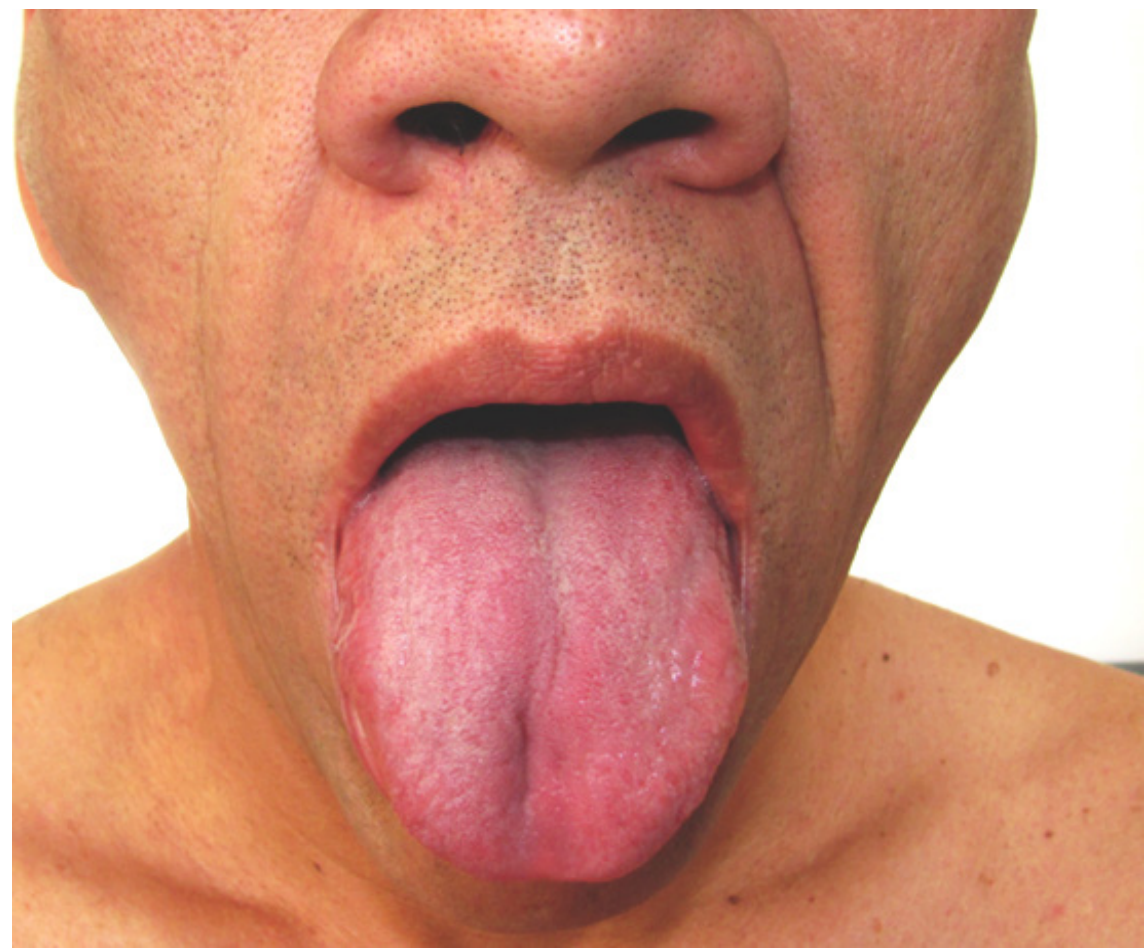

Figura 3. Macroglosia

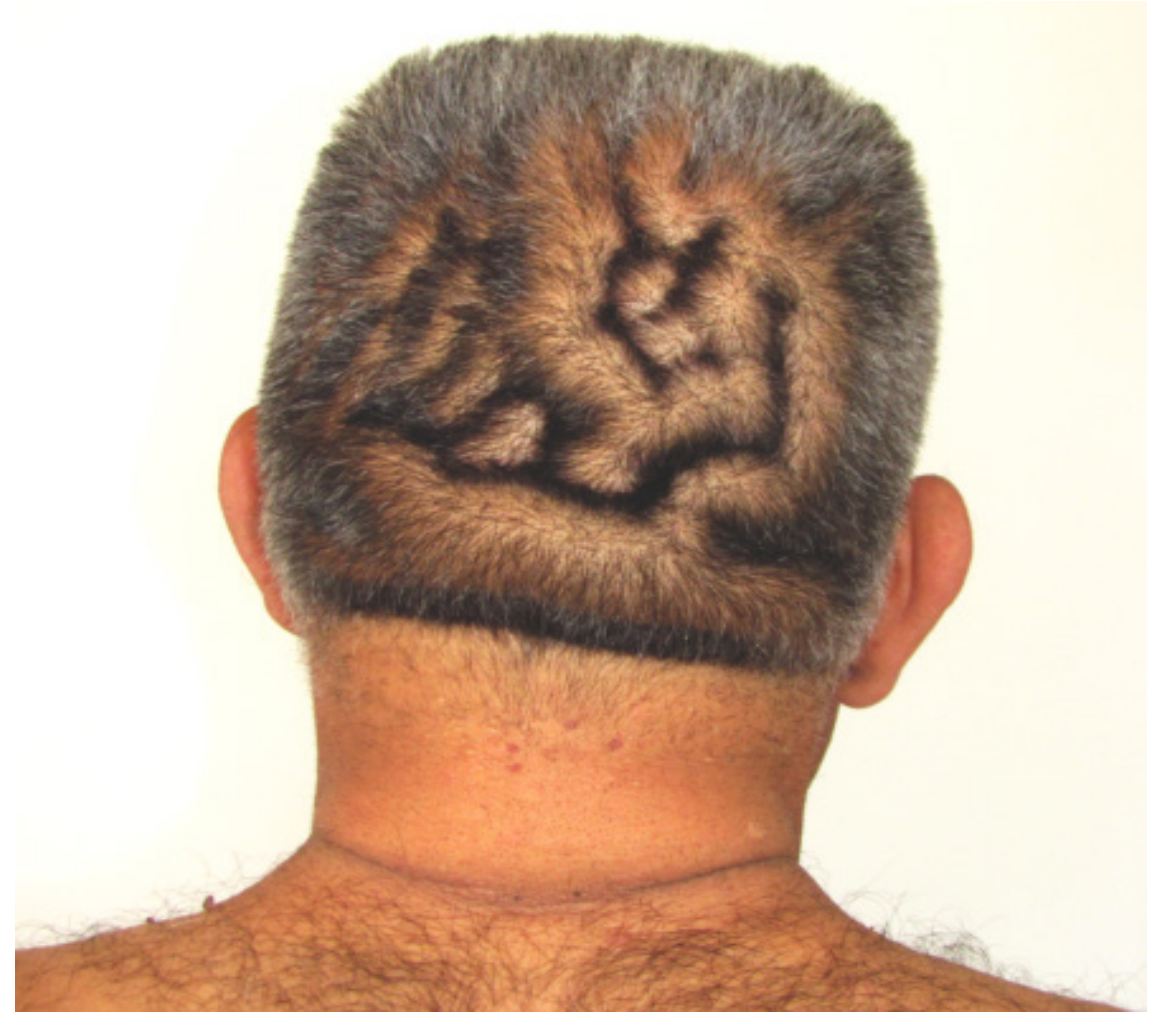

Figura 4. Cutis verticis gyrata 
Tabla 1. Manifestaciones cutáneas

\begin{tabular}{lcc} 
Alteraciones cután eas y de tejidos & $n$ & $\%$ \\
blandos & 17 & 77.3 \\
\hline Engrosamiento de la piel y del cuero cabelludo & 4 & 18.2 \\
\hline Cutis verticis gyrata & 4 & 18.2 \\
\hline Acantosis nigricans & 16 & 72.7 \\
\hline Fibromas laxos & 10 & 45.5 \\
\hline Hirsutismo o hipertricosis & 13 & 59.1 \\
\hline Hiperhidrosis & 2 & 9.1 \\
\hline Bromhidrosis & 10 & 45.5 \\
\hline Alteraciones ungulares & 8 & 36.4 \\
\hline Otras alteraciones (queratosis seborreicas, & 13 & 95.5 \\
queratosis actínicas y carcinoma basocelular) & 21 & 81.8 \\
\hline Agrandamiento distal (acral) & 18 & 63.6 \\
\hline Agrandamiento de nariz, orejas y labios & 14 & 68.2 \\
\hline Prominencia frontal & 15 & 68.2 \\
\hline Prognatismo & 15 & 54.5 \\
\hline Mala oclusión & 12 & 63.6 \\
\hline Macroglosia & 14 & 95.5 \\
\hline Alteraciones óseas & 15 & \\
\hline Agrandamiento distal (acral) & 15 & \\
\hline
\end{tabular}

Tabla 2. Enfermedades concomitantes

\begin{tabular}{lcc} 
Enfermedades & $n$ & $\%$ \\
\hline Hipertensión arterial sistémica & 11 & 50.0 \\
\hline Diabetes mellitus & 6 & 27.3 \\
\hline Dislipidemia & 10 & 45.5 \\
\hline Hipotiroidismo & 5 & 22.7 \\
\hline Cardiopatía & 4 & 18.2 \\
\hline Artropatía & 4 & 18.2 \\
\hline Pólipos intestinales & 3 & 13.6 \\
\hline Acroparestesias o síndrome del túnel carpiano & 12 & 54.5 \\
\hline Apnea del sueño & 5 & 22.7 \\
\hline Alteraciones sexuales o disminución de la libido & 12 & 54.5 \\
\hline Somnolencia o fatiga & 11 & 50.0 \\
\hline Cefalea & 10 & 45.5 \\
\hline Alteraciones visuales & 19 & 86.4
\end{tabular}


(área del triceps) con un grosor de la piel fue de $16 \mathrm{~mm}$ en promedio (rango de 7 a $29 \mathrm{~mm}$ ), y en el antebrazo, de $8,5 \mathrm{~mm}$ en promedio (rango de 3 a $16 \mathrm{~mm}$ ). Se comparó con un grupo control de 22 pacientes sanos con edades y sexo similares a los del grupo de estudio; en este grupo control, las medidas encontradas fueron de $12 \mathrm{~mm}$ en la zona posterior del brazo y de $6 \mathrm{~mm}$ en el antebrazo. Estas medidas, así como las de algunas partes corporales como cabeza, manos y pies, no mostraron una diferencia estadísticamente significativa (tabla 3).

\section{DISCUSIÓN}

La acromegalia, o gigantismo, es una enfermedad que resulta de la hipersecreción de hormona del crecimiento, usualmente por adenomas pituitarios. Afecta diversos órganos, generando efectos metabólicos y tróficos que se asocian con una mayor morbimortalidad en comparación con la de la población general. Esta enfermedad se denomina acromegalia cuando afecta a los adultos y gigantismo cuando los afectados son niños.

A pesar de su comienzo lento e insidioso y sus características clínicas inespecíficas, se han descrito manifestaciones dermatológicas que pueden llevar a un diagnóstico temprano, como: edema y consistencia pastosa con posterior engrosamiento de la piel, principalmente en cara, manos y pies; alteraciones de la sudoración (hiperhidrosis y bromhidrosis); piel grasosa con poros abiertos; aumento del tamaño del labio inferior y de la lengua; hipertricosis o hirsutismo; fibromas laxos o acrocordones múltiples y, en menor medida, acantosis nigricans ${ }^{(1,2,8)}$. El presente estudio arrojó hallazgos dermatológicos hasta en $95.5 \%$ de los casos analizados.

Estos hallazgos se producen por exceso de hormona de crecimiento o somatotropina y del factor de crecimiento similar a la insulina de tipo 1 (IGF-1) o somatomedina $\mathrm{C}$, los cuales actúan sobre las células de la piel y de los anexos, generando cúmulos de glucosaminoglucanos en la dermis, y estimulando constantemente los queratinocitos y fibroblastos.

Otras manifestaciones cutáneas descritas, son engrosamiento de las uñas, profundización de los pliegues en la cara, edema palpebral y cutis verticis gyrata. El acné no es una característica común ${ }^{(6,8-10)}$. En el presente estudio, se observaron cambios ungulares en 45.5 $\%$ de los casos y cutis verticis gyrata en $18.2 \%$, sin que se encontrara acné en ninguno de ellos.

Las manifestaciones clínicas no cutáneas descritas en la literatura incluyen cefalea y alteraciones visuales, y crecimiento distal, esquelético y de los tejidos blandos (crecimiento acral), con deformidades. Los cambios faciales observados fueron: prognatismo, mala oclusión, incisivos inferiores separados, prominencia frontal, hipertrofia de huesos nasales asociada al agrandamiento

Tabla 3. Mediciones corporales

\begin{tabular}{lccccc|} 
& N & Mínimo & Máximo & Media & Desv. típ. \\
$\begin{array}{l}\text { Piel grosor brazo - área del triceps }(\mathrm{mm}) \\
\text { en los casos }\end{array}$ & 22 & 7 & 29 & 16.00 & 6.901 \\
\hline Piel grosor antebrazo $(\mathrm{mm})$ en los casos & 22 & 3 & 16 & 8.55 & 3.188 \\
$\begin{array}{l}\text { Piel grosor brazo - área del triceps }(\mathrm{mm}) \\
\text { en controles }\end{array}$ & 22 & 6 & 20 & 12.86 & 4.027 \\
$\begin{array}{l}\text { Piel grosor antebrazo }(\mathrm{mm}) \text { en controles } \\
\text { P }\end{array}$ & 22 & 4 & 12 & 6.91 & 2.202
\end{tabular}

\begin{tabular}{lccccc}
\multicolumn{7}{c}{ Medidas } \\
Cabeza $(\mathrm{cm})$ & 22 & 51.0 & 65.0 & 58.273 & 29.949 \\
Manos $(\mathrm{cm})$ & 22 & 16.0 & 23.0 & 19.045 & 15.577 \\
Pies talla calzado & 22 & 31 & 44 & 39.45 & 2.824
\end{tabular}


de cabeza, manos y pies, que aumentan la talla de sombreros, anillos y zapatos, respectivamente ${ }^{(2,7,11)}$. En el presente grupo de pacientes, las manifestaciones clínicas incluyeron agrandamiento distal (acral) en 95.5 $\%$, prominencia frontal en $63.6 \%$, prognatismo y mala oclusión en $68.2 \%$, alteraciones óseas en $63.6 \%$, cefalea en $45.5 \%$ y alteraciones visuales en $86.4 \%$.

Otras alteraciones asociadas descritas son: artropatía, compromiso cardiaco como cardiomiopatía hipertrófica y arritmias, macroglosia con compromiso de las vías respiratorias superiores, y engrosamiento de la mucosa laríngea y las cuerdas vocales, que contribuye a producir cambios en la voz y apneas del sueño (12-15). También, son frecuentes las acroparestesias con síndrome de túnel carpiano asociado, la miopatía proximal y las mialgias ${ }^{(16)}$. En el presente estudio, se presentó cardiopatía en $18.2 \%$ de los casos, artropatía en $18.2 \%$, cambios en la voz en $36.4 \%$, alteraciones respiratorias y del sueño en $63.6 \%$, apnea del sueño en $22.7 \%$, somnolencia y fatiga en $50 \%$, y acroparestesias y síndrome del túnel carpiano en $54.5 \%$.

Hubo comorbilidades en $63.6 \%$ de los casos, como: hipertensión arterial en $50 \%$, diabetes mellitus en $27.3 \%$, dislipidemia en $45.5 \%$, hipotiroidismo en 22.7 $\%$, y alteraciones intestinales en $22.7 \%$, incluyendo pólipos intestinales en $13.6 \%$. Ningún paciente presentó cáncer de colon. En diversas publicaciones se describen alteraciones metabólicas como resistencia a la insulina y diabetes mellitus, dislipidemia e hipertensión arterial sistémica; además, pólipos o cáncer de colon, los cuales hacen que estos pacientes tengan una elevada morbimortalidad, dos a cuatro veces más alta que la de la población general ${ }^{(17-19)}$.

La hiperprolactinemia se asocia con tumores mixtos en $30 \%$ de los casos. En algunos de ellos, se pueden presentar deficiencias de glucocorticoides, esteroides sexuales $\mathrm{u}$ hormona tiroidea, que se manifiestan clínicamente como hipopituitarismo, amenorrea, hipogonadismo, disminución de la libido o impotencia $(2,17,20)$. En el presente estudio, se encontraron niveles de prolactina elevados en 16 pacientes, y hubo síntomas relacionados con alteraciones sexuales en 12 (54.5\%), disminución de la libido en 12 (54.5\%) e impotencia en $3(13.6 \%)$.

El diagnóstico clínico se confirma con pruebas de laboratorio que muestran aumento sérico del IGF-1 o somatomedina C, y con la medición de la hormona de crecimiento - somatotropina, dos horas después de la ingestión de 75 gramos de glucosa (prueba de tolerancia a la glucosa), confirmando el diagnóstico cuando no se produce supresión hormonal con disminución de su concentración a menos de 1 ng/ml. La se- creción de la somatotropina se produce en pulsos, por lo cual su concentración sérica basal es muy variable y, consecuentemente, no es confiable para el diagnóstico, por tal razón requiere confirmarse con la ausencia de supresión de la hormona mediante la prueba de tolerancia a la glucosa descrita previamente. Cuando estas pruebas se encuentran alteradas, se debe solicitar una RM de hipófisis para detectar microadenomas o macroadenomas de la hipófisis ${ }^{(21-23)}$. El presente grupo de estudio ya se encontraba en consulta de endocrinología con el diagnóstico de acromegalia o gigantismo que, en promedio, se había hecho 55.7 meses antes, y que se había confirmado con la medición de la somatomedina $\mathrm{C}$ y de la somatotropina. El hallazgo de un adenoma hipofisiario mediante resonancia magnética fue demostrado en todos ellos.

El tratamiento de estos pacientes requiere un enfoque multidisciplinario. En esta investigación, se encontró que el tratamiento médico incluyó lanreotide en el 81.8 $\%$ de los casos y octreotide en el $4.5 \%$, en el $13.6 \%$ no se formuló ninguna medicación. El $50 \%$ del grupo se sometió a cirugía y, el 36.4\%, a radioterapia.

Como en la mayoría de los casos informados se encuentra un adenoma hipofisiario, la resección quirúrgica con abordaje transesfenoidal es el tratamiento primario de elección, con el cual se obtiene mejoría clínica y de los exámenes paraclínicos. En 10 a 30 \% de los casos con tumores grandes, pueden presentarse recidivas.

Los análogos de la somatostatina hacen parte del tratamiento farmacológico, con dos medicamentos de acción prolongada, el octreotide y el lanreotide, ambos para uso intramuscular; este se reserva para pacientes con enfermedad residual posquirúrgica y para disminuir el tamaño de los macroadenomas antes de la cirugía; también, está indicado su uso en ancianos enfermos y en pacientes que rechazan la cirugía. En 75 $\%$ de los pacientes, los síntomas mejoran en varias semanas de tratamiento. Otras opciones farmacológicas son los antagonistas del receptor de la somatotropina, como el pegvisomant, para casos que no responden o no toleran el manejo con análogos de la somatostatina, y los agonistas de la dopamina, como la bromocriptina y la cabergolina, en tumores mixtos con aumento de los niveles de prolactina. La radioterapia hace parte del manejo coadyuvante en los que no mejoran con las medidas anteriores.

Con el tratamiento, las manifestaciones clínicas mejoran rápidamente; se puede presentar regresión completa o parcial en algunos casos ${ }^{(1,2,24-25)}$. Además, se deben tratar las comorbilidades, como hipertensión arterial sistémica, cardiopatía, diabetes, dislipidemia 
o alteraciones del colon, y la cirugía maxilofacial permite la reparación mandibular.

\section{CONCLUSIÓN}

La población analizada en este estudio no difiere de otras descritas en la literatura médica mundial; sin embargo, los hallazgos corroboran la gran frecuencia de las manifestaciones dermatológicas y las comorbilidades en estos pacientes, y su importancia al momento de hacer el enfoque clínico, ya que permiten sospechar la enfermedad y pueden llevar a un diagnóstico temprano que impacte positivamente en la morbimortalidad.

Los médicos deben estar atentos a estos hallazgos y sospechar la enfermedad al identificar los signos y síntomas descritos, así como las enfermedades sistémicas que pueden asociarse, para hacer el diagnóstico y brindar un tratamiento adecuado y oportuno.

\section{REFERENCIAS}

1. Adelman DT, Liebert K, Nachtigall LB, Lamerson M, Bakker B. Acromegaly: The disease, its impact on patients, and managing the burden of longterm treatment. Int J Gen Med. 2013;6:31-8.

2. Chanson P, Salenave S. Acromegaly. Orphanet J Rare Dis. 2008;3:1-17.

3. Holdaway IM, Rajasoorya C. Epidemiology of acromegaly. Pituitary. 1999;2:29-41.

4. Daly AF, Rixhon M, Adam C, Dempegioti A, Tichomirowa MA, Beckers A. High prevalence of pituitary adenomas: Across-sectional study in the province of Liege, Belgium. J Clin Endocrinol Metab. 2006;91:4769-75.

5. Ezzat S, Forster MJ, Berchtold P, Redelmeier DA, Boerlin V, Harris AG. Acromegaly. Clinical and biochemical features in 500 patients. Medicine (Baltimore). 1994;73:233-40.

6. Resende M, Bolfi F, dos Santos-Nunes V, AmanteMiot H. Prevalence of dermatologic disorders in 15 patients with acromegaly. An Bras Dermatol. 2012;87:166-8.

7. Kalus A, Chien A, Olerud J. Diabetes mellitus and other endocrine diseases. Acromegaly. In: Klaus Wolff, Lowell Goldsmith, Stephen Katz, Barbara Gilchrest, Amy Paller. Fitzpatrick's Dermatology in General Medicine. 7th. Edition. McGraw Hill companies. pag. 1461-84.

8. Ben-Shlomo A, Melmed S. Skin manifestations in acromegaly. Clin Dermatol. 2006;24:256-9.
9. Centurión SA, Schwartz RA. Cutaneous signs of acromegaly. Int J Dermatol. 2002,41:631-4.

10. Dahbar M, Danilowicz K,Malavela M, Velásquez D, Allevato M, Cabrera $\mathrm{H}$, et al. Manifestaciones cutáneas en la acromegalia. Dermatol Argent. 2009;15:186-90.

11. Lioté F, Orcel P. Osteoarticular disorders of endocrine origin. Best Pract Res Clin Rheumatol. 2000;14:251-76.

12. Lieberman SA, Björkengren AG, Hoffman AR. Rheumatologic and skeletal changes in acromegaly. Endocrinol Metab Clin North Am. 1992;21:61531.

13. Kamenicky P, Viengchareun S, Blanchard A, Meduri G, Zizzari P, Imbert-Teboul M, et al. Epithelial sodium channel is a key mediator of growth hormone-induced sodium retention in acromegaly. Endocrinology. 2008;149:3294-305.

14. Mosca S, Paolillo S, Colao A, Bossone E, Cittadini A, IudiceFL, et al. Cardiovascular involvement in patients affected by acromegaly: An appraisal. Int J Cardiol. 2013;167:1712-8.

15. Attal P, Chanson P. Endocrine aspects of obstructive sleep apnea. J Clin Endocrinol Metab. 2010;95:483-95.

16. Jenkins PJ, Sohaib SA, Akker S, Phillips RR, Spillane K, Wass JA, et al. The pathology of median neuropathy in acromegaly. Ann Intern Med. 2000;133:197-201.

17. Ruiz R, Durán EG, Arellano SA, Sánchez V, Moreno OT, Mendoza F. Acromegalia. Med Int Mex. 2009;25:468-80.

18. Berg C, Petersenn S, Lahner H, Herrmann BL, Buchfelder M, Droste M, et al. Cardiovascular risk factors in patients with uncontrolled and long-term acromegaly: Comparison with matched data from the general population and the effect of disease control. J Clin Endocrinol Metab. 2010;95:3648-56.

19. Dutta P, Bhansali A, Vaiphei K, Dutta U, Ravi Kumar P, Masoodi S, et al. Colonic neoplasia in acromegaly: Increased proliferation or deceased apoptosis? Pituitary. 2012;15:166-73.

20. Andersen M. Management of endocrine disease: GH excess: Diagnosis and medical therapy. Eur J Endocrinol. 2013;170:31-41.

21. Rúa C, Latorre G, Campuzano G. Diagnóstico de acromegalia. Medicina y Laboratorio. 2011;17:511531.

22. Kannan S, Kennedy L. Diagnosis of acromegaly: State of the art. Expert Opin Med Diagn. 2013;7:443-53. 
23. Scacchi M, Cavagnini F. Acromegaly. Pituitary. 2006;9:297-303.

24. Bianchi A, Valentini F, Iuorio R, Poggi M, Baldelli $\mathrm{R}$, PasseriM, et al. Long-term treatment of somatostatin analog-refractory growth hormone-secreting pituitary tumors with pegvisomant alone or combined with long-acting somatostatin analogs: A retrospective analysis of clinical practice and outcomes. J ExpClin Cancer Res. 2013;32:1-11.

25. Pita-Gutiérrez F, Pertega-Díaz S, Pita-Fernández $\mathrm{S}$, Pena L, Lugo G, Sangiao-Alvarellos S, et al. Place of preoperative treatment of acromegaly with somatostatin analog on surgical outcome: A systematic review and meta-analysis. PLoS One. 2013;8:e61523. 